\title{
Assessment of the impact of an exercise program on the physical and functional capacity in patients with autosomal recessive spastic ataxia of Charlevoix-Saguenay: An exploratory study
}

\author{
Olivier Audet ${ }^{1}$, Hung Tien Bui ${ }^{2}$, Maxime Allisse ${ }^{3}$, Alain-Steve Comtois ${ }^{4}$, Mario Leone ${ }^{3, *}$ \\ 'Deparment of Kinesiology, Université Laval, Québec, Canada; \\ ${ }^{2}$ Department of Applied Sciences, Université du Québec à Chicoutimi, Saguenay, Canada; \\ ${ }^{3}$ Department of Health Sciences, Université du Québec à Chicoutimi, Saguenay, Canada; \\ ${ }^{4}$ Department of Exercise Sciences, Université du Québec à Montréal, Montréal, Canada.
}

\begin{abstract}
Summary Autosomal recessive spastic ataxia of Charlevoix-Saguenay (ARSACS) is a neuromuscular disorder caused by the mutation of the SACS gene. Clinical symptoms of this disease include progressive ataxia, spasticity, and peripheral neuropathy. Similar to other neuromuscular disorders, these patients are prone to physical deconditioning which may lead to a loss of functional capacity. This paper aims to evaluate the impact of a training program on the physical fitness and the functional capacity of ARSACS patients. Twelve patients (age: $28.1 \pm 8.2$ years) participated in this study. They followed an eight-week training program including physical activities, strength-power and aerobic training. Compared to the initial evaluation, measures of physical fitness and functional capacity were significantly improved $(p \leq 0.05)$ for 11 of the 12 tests. Positive gains were also observed for fall frequency and for upper-limb incoordination. This paper supports the importance of a training program for ARSACS patients in order to improve their quality of life. Through these types of interventions, it may be possible to slow down the progression of the disease and help maintain functional capacity.
\end{abstract}

Keywords: ARSACS, Ataxia, exercise training, functional capacity, upper-limb incoordination

\section{Introduction}

Autosomal recessive spastic ataxia of CharlevoixSaguenay (ARSACS) is a rare disease which has been documented for the first time by Bouchard et al. in 1978 (1). The discovery of the disease's etiology is recent, and the pathology basis and the mechanical physiopathology are not fully understood. Located on chromosome 13, a mutation of the SACS gene hinders the proper production of the sacsin protein $(4,579-a)$, which is the cause of ARSACS. This disruption leads

Released online in J-STAGE as advance publication August 15, 2018.

*Address correspondence to:

Dr. Mario Leone, Department of Health, Kinesiology Division, Université du Québec à Chicoutimi, 555 boulevard de l'Université, Saguenay (Québec), G7H 2B1, Canada.

E-mail: mario.leone@uqac.ca to an abnormal dendrite morphology which affects the transfer of nerve impulses and consequently leads to the death of Purkinje cells in the cerebellum (2). The main clinical symptoms associated to this disease are progressive ataxia, spasticity and peripheral neuropathy (3). ARSACS is a hereditary disease that is specific to the Charlevoix and Saguenay-Lac-Saint-Jean (CSLSJ) regions $(4,5)$. Like other recessive diseases, ARSACS has a high prevalence in these regions while it is rare worldwide. Nevertheless, some cases were observed in countries like Japan, Italy, Tunisia and Turkey (O).

ARSACS, as with other neuromuscular/neurological diseases, generally brings patients to be less active and causes physical deconditioning $(7,8)$. This could result in an important decrease in functional capacity and could even lead to a premature loss of autonomy. Actual best practice management of ARSACS patients is mostly palliative and is not sufficient to maintain their quality of life.

Recent research demonstrated that physical activity 
and training have a positive impact on patients with pathologies similar to ARSACS. More precisely, results show a positive association between strength training and certain symptoms of neuromuscular disorder (NMD) and neurological conditions (9-21). Furthermore, as demonstrated by several authors $(8,12,17,22-25)$, patients affected by certain NMD can even improve their locomotor skills following specific training programs. However, to our knowledge, the impact of physical training has not yet been established with ARSACS patients.

Thus, the objective of this paper is to evaluate the impact of an exercise program on the physical fitness and the functional capacity of patients affected by ARSACS. Based on results from existing NMD studies, our hypothesis is that the exercise program will have a positive impact on ARSACS patients by increasing their physical fitness level and, consequently, their functional capacity.

\section{Materials and Methods}

\subsection{Participants}

Twelve early-onset ARSACS patients (5 males and 7 females) aged between $17-45$ years (28.1 \pm 8.2 years) participated in this study. They were recruited from a list provided by Muscular Dystrophy Canada. Traditionally, the severity of the disease in ARSACS patients is typically evaluated in clinic based on walking mobility $(3,26)$ rather than using a standardized scale. While several ataxia severity scales exist, such as the Scale for the Assessment and Rating Ataxia - SARA (27) and the International Cooperative Ataxia Rating Scale - ICARS (28), they do not consider all three components of the disease (pyramidal, cerebellar and neuropathic) in order to provide a more complete picture. In addition, none of these scales have been validated in patients with ARSACS. Even though it is conceptually possible to perform a combination of these severity scales, it is not a viable option due to large number of items that need to be evaluated. Participants were thus classified by severity according to their ability to walk: light ( $n$ $=5$, walking without aid), moderate $(n=3$, walking aid required) and severe ( $n=4$, wheelchair-bound).

All participants were at least sixteen years of age, which is the legal age at which a person can give their consent to participate in a study according to the applicable local laws. To be included in the study, participants must have been diagnosed with ARSACS through genetic testing. The only exclusion criterion was to have received medical contraindications for the practice of physical activities. All participants gave their informed written consent to participate in this research, which was approved by the University Ethics Committee Board (No.602.381.01).

\subsection{Testing protocol}

During the first week, all participants were assessed using 5 strength and 7 functional tests, for a total of 12 assessment tests. Assessments were separated into two 90-minute sessions to ensure optimal performance. There were 12 stations, each corresponding to a different test, and participants went through each one sequentially. Between each station, there was a two-minute rest period. This same procedure was also followed during the last (ninth) week of the program.

\subsection{Physical fitness tests}

Assessment of physical fitness was performed using conventional weight training equipment. During the first week, a maximum strength test was administered for each of the 5 strength exercises (Figure 1) identified using the one-repetition maximal load (1RM). Since it is difficult and time-consuming to precisely establish the maximum load that can only be lifted once, the maximum load was established from the 1RM table suggested by Baechle et al. (29). In order to estimate the $1 \mathrm{RM}$, the maximum number of repetitions accepted was 6 without which the participant had to start again with a load increased by $20 \%$.

To assess abdominal strength, the participant was seated in front of the pulley and held the rope handle on his chest. The aim is to lift the heaviest possible load by bending the trunk forward to an angle of $90^{\circ}$ (Figure 1A).

Upper-limb strength (bench press) was measured while the participant was sitting. The arms are bent at $90^{\circ}$ at shoulder height. The participant would then lift the load with a complete extension of the arms (Figure 1B).

The biceps curl was assessed while the participant was seated with elbows supported in extension $\left(180^{\circ}\right)$ and hands in supine position. The participant would then lift the load (flexion of the elbows) with both arms in order to reach a vertical position with arms bent at $50^{\circ}$ $60^{\circ}$ (Figure 1C).

Maximal muscle strength of the quadriceps was measured on a knee extension device (Figure 1D). While the participant was seated, their feet were placed under the cushioned roll while the thigh-calf angle is at $90^{\circ}$. The load was lifted with a full extension of the knees which represents $180^{\circ}$.

Finally, pectoral strength was assessed using a pectoral fly device (Figure 1E). In the sitting position, the participant had their arms open so that they form an angle of $180^{\circ}$ while the elbows are bent at $90^{\circ}$. From this position, the elbows were bent forward by a movement towards the median plane (adduction).

\subsection{Functional capacity tests}

Functional capacity was evaluated using parts of the testing protocol proposed by Kalinova and Leone (30) 


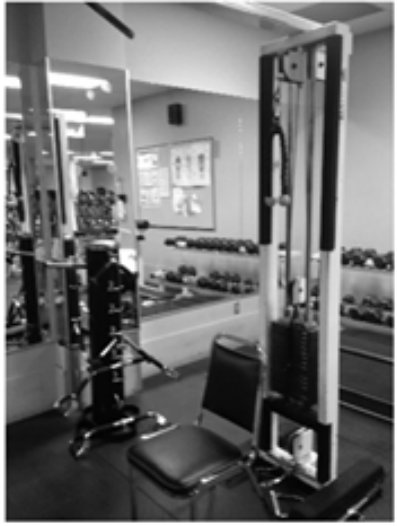

(A)

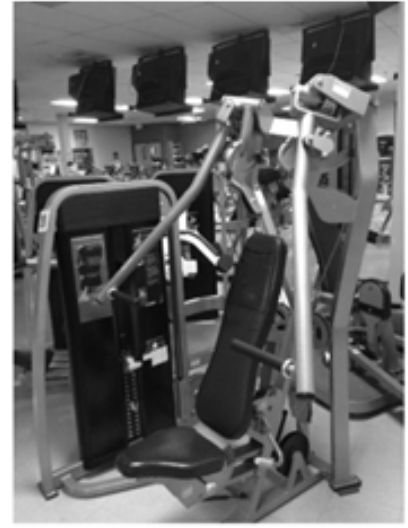

(B)

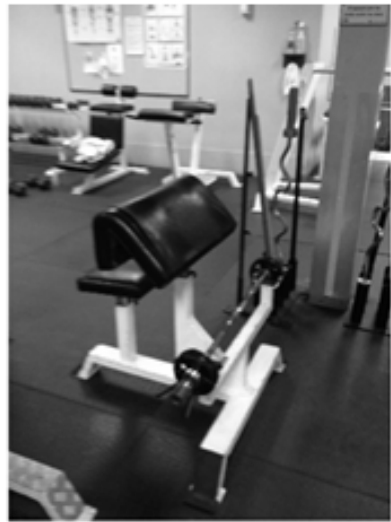

(C)

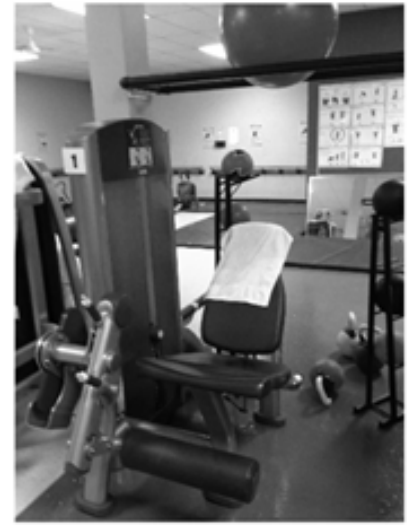

(D)

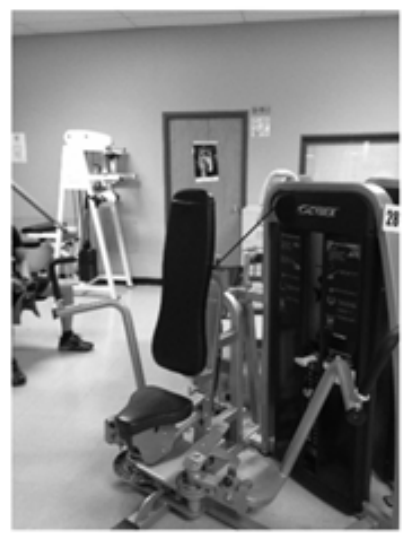

(E)

Figure 1. Pictures of the training devices used with ARSACS patients. (A) Abdominal strength; (B) Bench press; (C) Biceps curls; (D) Knee extension; (E) Pectoral fly.

which include one-hand plate tapping, sit-and-stand, six-minute walk test and the hand grip strength test (Table 1). In addition, a Swiss ball was used to assess trunk stability/balance, a bench press test was used to evaluate muscular endurance of the upper-limbs, a $10 \mathrm{~m}$-walk test assessed the maximal walking speed and a questionnaire was used to evaluate the frequency of falls within the previous week.

The one-hand plate tapping test, which assesses the speed of the upper-limbs, consisted of moving laterally the dominant hand as quickly as possible by touching alternatively each of the two circles which were separated by a distance of $60 \mathrm{~cm}$. The result is expressed as the total number of hits within 20 seconds $\left(\mathrm{nb} \cdot 20 \mathrm{~s}^{-1}\right)$.

The sit-and-stand test requires participants to sit and rise from a standard chair without using their arms. The test ended after 20 seconds during which the number of sit-and-stand was counted (nb·20s $\left.{ }^{-1}\right)$.

The bench press test allows for the measurement of the participant's capacity to move a load that represents $40 \%$ of $1 \mathrm{RM}$ as many times as possible within 20 seconds $\left(\mathrm{nb} \cdot 20 \mathrm{~s}^{-1}\right)$. The total number of repetitions was noted.

Several reliable test batteries, such as the Berg
Table 1. Description of factors and variables used to assess ARSACS patients for functional capacity and physical fitness evaluations

\begin{tabular}{|c|c|}
\hline Factors & Variables \\
\hline \multicolumn{2}{|l|}{ Functional capacity variables } \\
\hline Speed (upper limbs) & 1- One-hand plate tapping $\left(\mathrm{nb} \cdot 20 \mathrm{~s}^{-1}\right)$ \\
\hline Speed (lower limbs) & 2- Sit-and-stand $\left(\mathrm{nb} \cdot 20 \mathrm{~s}^{-1}\right)$ \\
\hline Endurance (upper limbs) & 3- Bench press $\left(\mathrm{nb} \cdot 20 \mathrm{~s}^{-1}\right)$ \\
\hline Balance & 4- Swiss ball balance test (s) \\
\hline Grip strength & 5- Hand grip strength $(\mathrm{kg})$ \\
\hline Functional capacity to walk & 6- Six-minute walk test (m) \\
\hline Walking maximal speed & 7- 10m-walk test (s) \\
\hline \multicolumn{2}{|l|}{ Physical fitness variables } \\
\hline Maximum muscle strength & 8- Abdominal strength (kg) \\
\hline & 9- Bench press (kg) \\
\hline & 10- Biceps curls (kg) \\
\hline & 11- Knee extension (kg) \\
\hline & 12- Pectoral fly (kg) \\
\hline
\end{tabular}

Number of repetitions (nb); Kilogram (kg); Second (s); Watts (w); Meter (m).

balance scale, can be used to evaluate balance and/or stability in the general population. However, since the majority of the participants are either wheelchair bound 
Table 2. Specific parameters of the strength and the power training

\begin{tabular}{|c|c|c|c|}
\hline Items & Parameter & Strength training & Power training \\
\hline Week 1 & Initial evaluation & - & - \\
\hline \multirow[t]{4}{*}{ Week 2} & Set & 1 & 1 \\
\hline & Repetition & $3-6$ & $6-8$ \\
\hline & Rest period & 2 minutes & 1 minute \\
\hline & Load & $80 \%$ of $1 \mathrm{RM}$ & $40 \%$ of $1 \mathrm{RM}$ \\
\hline \multirow[t]{4}{*}{ Weeks 3 to 8} & Set & 2 & 1 \\
\hline & Repetition & $3-6$ & $6-8$ \\
\hline & Rest period & 2 minutes & 1 minute \\
\hline & Load & $80 \%$ of $1 \mathrm{RM}$ & $40 \%$ of $1 \mathrm{RM}$ \\
\hline
\end{tabular}

$1 \mathrm{RM}=$ One repetition maximum.

Table 3. Specific parameters of the aerobic training

\begin{tabular}{|c|c|c|c|}
\hline Week & $\begin{array}{c}\text { Session } 1 \\
\text { Set } \times[\text { minutes ran }(r) \text { and/or walked }(w)]\end{array}$ & $\begin{array}{c}\text { Session } 2 \\
\text { Set } \times[\text { minutes ran }(\mathrm{r}) \text { and/or walked }(\mathrm{w})]\end{array}$ & Weekly volume (min) \\
\hline 1 & Initial evaluation & Initial evaluation & Initial evaluation \\
\hline 2 & $1 \times(15 r)$ & $1 \times(15 r)$ & 30 \\
\hline 3 & $3 \times(4 \mathrm{r} / 1 \mathrm{w})$ & $8 \times(1 r / 1 w)$ & 31 \\
\hline 4 & $4 \times(4 r / 1 w)$ & $10 \times(1 \mathrm{r} / 1 \mathrm{w})$ & 40 \\
\hline 5 & $1 \times 21 r$ & $11 \times(1 \mathrm{r} / 1 \mathrm{w})$ & 43 \\
\hline 6 & $5 \times(4 r / 1 w)$ & $12 \times(1 \mathrm{r} / 1 \mathrm{w})$ & 49 \\
\hline 7 & $4 \times(5 r / 1 w)$ & $13 \times(1 \mathrm{r} / 1 \mathrm{w})$ & 50 \\
\hline 8 & $4 \times(6 r / 1 w)$ & $13 \times(1 \mathrm{r} / 1 \mathrm{w})$ & 54 \\
\hline
\end{tabular}

or require a walking aid, most items in the balance scale would not be feasible. Therefore, the Swiss ball trunk stability test with eyes open, proposed by Noehren and colleagues, was used (31). This test determines the participant's capacity to maintain their stability/balance in a seated position while having one foot in contact with the ground. The final score corresponds to the length of time (s) that the participant was able to remain seated on the Swiss ball without using their hands or the free leg. The test can also end if the participant succeeds to maintain balance for one minute.

The hand grip test (manual dynamometer; Takei Kiki Kogyo model tk-1210, Japan) allows for the measurement of the maximal strength developed by the finger joints and the forearm. Two trials for each hand were allowed and the sum of the best score for each hand was noted $(\mathrm{kg})$.

The six-minute walk test is a popular method of assessing the walking ability of individuals affected by different conditions. This test was administered according to the procedure described by American Association of Cardiovascular and Pulmonary Rehabilitation (32) for patients who are able to walk with or without walking aid. Wheelchair patients performed the test using their own wheelchair. The results are expressed as the distance (m) traveled in 6 minutes.

The $10 \mathrm{~m}$-walk test consists in traveling a distance of $10 \mathrm{~m}$ as fast as possible (33). The original procedure was slightly modified by adjusting the distance to $10 \mathrm{~m}$ instead of the more common typical $7.62 \mathrm{~m}$ distance. Patients were allowed to use their wheelchairs or walking aid when needed. The results of the test correspond to the time (s) required to complete the total $10 \mathrm{~m}$ distance.

In addition, upper-limb coordination was also assessed using a computerized version of the Archimedes spiral test (34). Results were extracted and frequency analysis was performed in order to examine smoothness of movement, which is a good indicator of coordination. Finally, participants completed a questionnaire aiming to assess, on a scale from 1 (never) to 10 (very often), the impact of the program on the frequency of their falls (pre $v s$. post-training).

The eight-week training program consists of two 90-minute sessions every week. The content of each training session depends on the degree of severity of the disease. For the lightly and moderately affected groups, the sessions were divided into three parts: $i) 30$ minutes of physical activities or sports (volleyball, badminton, etc.); ii) 30 minutes of strength and power training using resistance training devices; iii) 30 minutes of aerobic fitness training on an indoor track or an aerobic training machine (treadmill, bicycle, rowing machine). The group consisting of severely affected patients did not participate in the physical activities and sports portion of the training. Instead, their training only focused on strength and power exercises (45 minutes) and aerobic fitness (45 minutes). This ensures that both groups have the same training volume. The specific parameters of the training program are presented in Table 2 (strength and power exercises) and Table 3 (aerobic fitness). Each training session was supervised by two qualified kinesiologists. 
Table 4. ARSACS patient performance on functional capacity evaluations

\begin{tabular}{|c|c|c|c|c|}
\hline Variables & Pre-training $(n=12)$ & Post-training $(n=12)$ & $p$-values & Cohen's $d$ Effect Size \\
\hline 1. Plate tapping $\left(\mathrm{nb} \cdot 20 \mathrm{~s}^{-1}\right)$ & $49.8 \pm 14.1$ & $56.0 \pm 13.6$ & 0.003 & 0.45 \\
\hline 2. Sit-and-stand $\left(\mathrm{nb} \cdot 20 \mathrm{~s}^{-1}\right)$ & $8.3 \pm 3.0$ & $10.1 \pm 2.2$ & 0.007 & 0.68 \\
\hline 3. Bench press $\left(n b \cdot 20 s^{-1}\right)$ & $16.8 \pm 9.2$ & $21.5 \pm 9.3$ & 0.003 & 0.51 \\
\hline 4. Swiss ball balance test (s) & $4.3 \pm 5.8$ & $6.7 \pm 7.9$ & 0.003 & 0.35 \\
\hline 5. Hand grip strength $(\mathrm{kg})$ & $54.8 \pm 20.8$ & $60.1 \pm 21.5$ & 0.005 & 0.25 \\
\hline 6. Six-minute walk test $(\mathrm{m})$ & $318.7 \pm 120.4$ & $375.6 \pm 131.6$ & 0.008 & 0.45 \\
\hline 7. $10 \mathrm{~m}$-walk test (s) & $9.1 \pm 6.4$ & $8.1 \pm 4.6$ & 0.117 & 0.18 \\
\hline
\end{tabular}

Descriptive statistics are presented as means and standard deviation (mean $\pm \mathrm{SD}$ ). Bold characters indicate statistical significance at $p<0.05$. Cohen's $d$ coefficient: 0.20 small; 0.50 medium; 0.80 large. Number of repetitions (nb).

Table 5. ARSACS patient performance on physical fitness evaluations

\begin{tabular}{|c|c|c|c|c|}
\hline Variables & Pre-training $(n=13)$ & Post-training $(n=13)$ & $p$-values & Cohen's $d$ Effect Size \\
\hline \multicolumn{5}{|l|}{ Physical fitness } \\
\hline 1. Abdominal strength $(\mathrm{kg})$ & $31.4 \pm 7.9$ & $44.1 \pm 11.7$ & 0.003 & 1.10 \\
\hline 2. Bench press $(\mathrm{kg})$ & $56.0 \pm 27.4$ & $76.1 \pm 41.7$ & 0.003 & 0.58 \\
\hline 3. Biceps curls $(\mathrm{kg})$ & $21.2 \pm 8.7$ & $26.8 \pm 8.0$ & 0.005 & 0.67 \\
\hline 4. Knee extension $(\mathrm{kg})$ & $58.8 \pm 20.9$ & $75.5 \pm 26.9$ & 0.002 & 0.69 \\
\hline 5. Pectoral fly $(\mathrm{kg})$ & $41.2 \pm 18.4$ & $49.8 \pm 19.6$ & 0.003 & 0.45 \\
\hline \multicolumn{5}{|l|}{ Fall frequency } \\
\hline 1. Number of falls (nb/week) & $2.9 \pm 1.7$ & $1.7 \pm 0.8$ & 0.015 & 0.90 \\
\hline
\end{tabular}

Descriptive statistics are presented as means and standard deviation (mean $\pm \mathrm{SD}$ ). Bold characters indicate statistical significance at $p<0.05$. Cohen's $d$ coefficient: 0.20 small; 0.50 medium; 0.80 large.

\subsection{Statistical analysis}

Participants' physical fitness and functional capacity measurements are reported as mean \pm standard deviation (SD). Because of the small sample size and violation of normality test assumptions, nonparametric pairedsamples Wilcoxon signed-rank tests were used to analyze the differences between pre and post-training evaluations. Cohen's $d$ coefficients were calculated in order to estimate the effect sizes (ES). All statistical analyses were performed using SPSS version 24. Finally, values of $p \leq 0.05$ were established as significant.

\section{Results}

\subsection{Functional capacity and physical fitness evaluations}

Table 4 and Table 5 present the results of the physical fitness and functional capacity tests. They show that most tests (11 out of 12) display statistically significant improvements between the initial and the final evaluations. When considering functional capacity, 6 out of 7 tests showed significant differences (one-hand plate tapping, sit-and-stand test, bench press (muscular endurance), Swiss ball balance test, hand grip strength, six-min walk test). The majority of functional tests showed ES values ranging from moderate to large (Table 4). Results obtained for the $10 \mathrm{~m}$-walk test revealed no significant improvement. With respect to physical fitness evaluations, all 5 tests showed significant differences between pre and post-training periods (Table 5). Their calculated ES ranged from moderate to very high.

\subsection{Questionnaire on the frequency of falls}

Answers to the questionnaire on the frequency of falls (Table 5) reveal that there is a significant difference $(p=0.04)$ between pre-training scores (2.2) and posttraining scores (3.2). The calculated ES is considered moderate $(0.5)$.

\subsection{Upper-limb coordination}

Figures $2 \mathrm{~A}, 2 \mathrm{~B}$ and $2 \mathrm{C}$ show the superimposed spiral traces drawn by all participants. Figure $2 \mathrm{~A}$ shows the results of spiral traces performed by ARSACS patients before training whereas Figure 2B shows the results after the eight-week program. For the purpose of comparison, Figure $2 \mathrm{C}$ presents spiral traces produced by 36 healthy age-matched participants. It shows a spiral shape of a certain thickness that illustrates that even healthy participants deviate a little from the reference.

Compared to Figure 2C, the spiral traces from Figure $2 \mathrm{~A}$ were markedly entangled. After an eightweek training program, the spiral traces produced by the participants became visibly less entangled (Figure 2B). Pre and post-training results of frequency analysis were compared and $p$-values for each frequency are plotted in Figure 2D. The figure indicates that frequency 


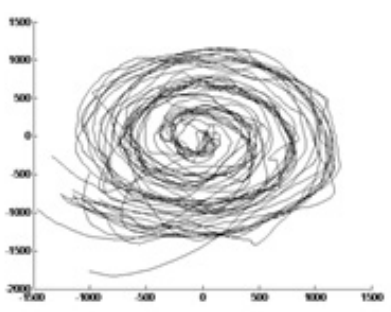

(A)

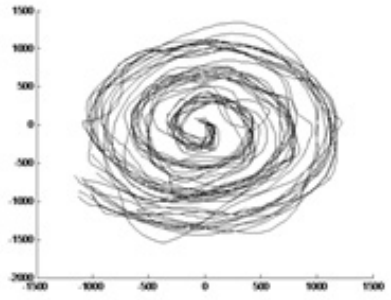

(B)

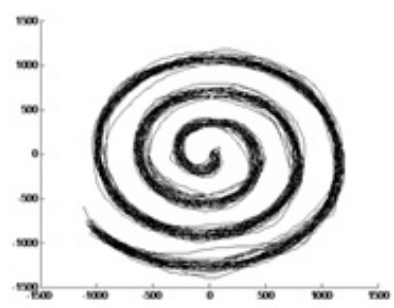

(C)

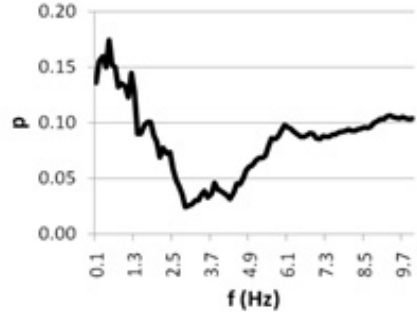

(D)

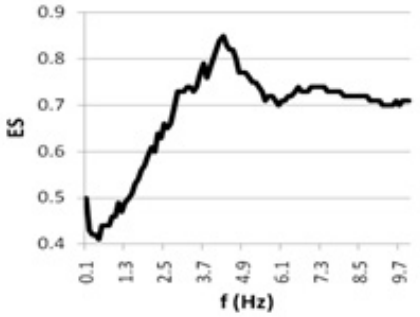

(E)

Figure 2. Comparison between ARSACS patients before (A) and after training (B); Spiral trace performed by healthy participants (C); p values related to the statistical significance of differences between trained and untrained ARSACS patients (D); ES coefficients related to the clinical differences between trained and untrained ARSACS patients (E).

components between $2.5 \mathrm{~Hz}$ and $4.7 \mathrm{~Hz}$ showed significant changes after the exercise program. Figure $2 \mathrm{D}$ shows the result of the comparison ( $p$ value) for each frequency between pre and post-training. Beyond the statistical significance, the values of the ES coefficients highlight the clinical importance of the changes in the level of ataxia (Figure 2E). At $\mathrm{f}=4.3 \mathrm{~Hz}$, the ES coefficients reached a maximum value of 0.85 , indicating a high clinical impact.

\section{Discussion}

Results of this study support the hypothesis that an eight-week specific training program improves physical fitness and functional capacity of ARSACS patients. This demonstration is similar to what was observed in the case of neuromuscular $(9,18)$ or neurological conditions $(10,19)$. This study is, to our knowledge, the first one to assess the impact of an exercise program on the physical fitness and functional capacity of patients affected by ARSACS.

\subsection{Impact of resistance training on muscle strength and functional capacity}

The strength-power training program generated positive adaptations in a number of muscular groups. In addition, according to the evaluation, functional capacity was also improved. This is interesting as there was no specific exercise aimed at improving functional capacity. This can be partially explained by Bohannon's theory (35) which hypothesizes that a gain in strength improves the neuronal condition between the agonist and the antagonist muscles, which enhances the execution of certain functional tasks.

\subsection{Changes in walking ability and balance}

The participants recruited in this study were initially considered inactive. As the study progressed, their walking ability improved possibly due to the development of their aerobic capacity, lower limb strength and balance. The results of the questionnaire revealed that participants fell less often at the end of the eight-week training program. This outcome may be attributed to benefits obtained from the strength-power training program. Indeed, strength and power have often been associated to fall prevention and the results from this study support those findings (36-38).

Comparison between pre and post-training for the $10 \mathrm{~m}$-walk test showed no significant differences. As well, the ES value was also modest. Several reasons may explain this result. The most likely explanation is that this test was not well adapted to the chosen population. In fact, ARSACS patients in our sample formed a very heterogeneous group with some who are able to walk and others who are wheelchair-bound. When the four wheelchair patients were excluded from the analysis, the $10 \mathrm{~m}$-walk test became statistically significant ( $p=$ 0.05). Despite this result, the ES values remain fairly modest, with values below 0.25 . Indeed, steady-state aerobic training is not known to contribute to improve the performance in a walking speed task. In addition, strength and power training of leg muscles, without specific sprint training, will likely not improve the performance of the $10 \mathrm{~m}$-walk test. 


\subsection{Impact of upper-limb strength training program on movement incoordination}

Upper-limb coordination of ARSACS patients, as measured by the Archimedes spiral test, showed statistical and clinical improvement after the eight-week training program. These changes can be observed when visually comparing the superimposed spiral traces pre and post training. In addition, these visual differences also reflect changes in frequency analysis results. Indeed, analysis was performed on a large range of frequencies and results showed significant differences on certain frequency bands between pre and posttraining. These results support previous observations that certain frequency bands are more sensitive than others in evaluating incoordination (34). Indeed, the work of Bui et al. has shown that frequency analysis is a very sensitive tool to discriminate healthy participants from ARSACS patients, particularly between $1.2 \mathrm{~Hz}$ and 1.7 Hz. In this study, after an eight-week training period, the ARSACS patients significantly improved their frequency analysis scores between frequency bands of $2.5 \mathrm{~Hz}$ and 4.7 Hz. This difference in frequency bands suggests that the training program helps improve coordination, as measured by the spiral test, but does not necessarily improve frequency components that distinguish ARSACS participants from healthy ones. While there were no coordination-specific exercises performed during the program, the authors believe that physical fitness training was a major contributor in this positive change. Indeed, a better stability of the shoulder, due to improved shoulder girdle strength, may be responsible for coordination gain and decreased symptoms of ataxia.

\subsection{Study limitations}

Due to the fact that ARSACS is a rare disease, the number of participants was limited to twelve. While it may seem like a small number, it is important to state that there are only about 300 known cases of ARSACS in the CSLSJ region. In addition, the problem with limited participation is also common in others studies with rare NMD (39). A second limitation of this study is that the training period consisted of eight weeks and that no conclusions can be drawn on its impact in the long-term. Finally, the absence of a control group can be considered a limitation. However, since the objective of this study was to examine the response to physical exercise, pre and post-training evaluations seem to indicate promising results on their physical autonomy.

\section{Conclusion}

This study presents an eight-week exercise program and its effect on the physical fitness and functional capacity of people affected by ARSACS. To our knowledge, this is the first study that evaluates the impact of training on the ARSACS population. While this is still a preliminary study, our results suggest that physical training does not have a deleterious effect on their musculoskeletal and/or cardiorespiratory functions. In fact, results of pre and post-training assessments show that most tests (11 out of 12) presented significant improvement. For instance, maximal strength evaluations suggest that muscular capacity can be improved following a resistance training program. These results also seem to contribute to improving functional capacity even though no specific exercises were prescribed, which is in accordance with Bohannon's theory. In addition, since ES values range from moderate to high for most variables studied, these changes seem to suggest that important improvements can be obtained from a patient's clinical perspective in day-to-day activities. Indeed, as indicated by the significant reduction in the frequency of falls and by the large value of the effect size, these results can be seen as a global indicator of the potential benefits of this type of intervention.

The overall positive change observed in these patients provides an encouraging sign that it is possible to help ARSACS patients maintain or regain their autonomy. This can have a positive impact both on an economic and a social level.

\section{Acknowledgements}

The authors wish to thank Muscular Dystrophy Canada and Ataxia Charlevoix-Saguenay for their time, materials and the funding which allowed us to conduct this research. We would also like to thank all participants who volunteered to participate in this study.

\section{References}

1. Bouchard JP, Barbeau A, Bouchard R, Bouchard RW. Autosomal recessive spastic ataxia of CharlevoixSaguenay. Can J Neurol Sci. 1978; 5:61-69.

2. Girard M, Larivière R, Parfitt DA, et al. Mitochondrial dysfunction and Purkinje cell loss in autosomal recessive spastic ataxia of Charlevoix-Saguenay (ARSACS). Proc Natl Acad Sci USA. 2012; 109:1661-1666.

3. Duquette A, Brais B, Bouchard JP, Mathieu J. Clinical presentation and early evolution of spastic ataxia of charlevoix-saguenay. Mov Disord. 2013; 28:2011-2014.

4. Heyer E, Tremblay M, Desjardins B. Seventeenth-century European origins of hereditary diseases in the Saguenay population (Quebec, Canada). Hum Biol. 1997; 69:209225.

5. Thiffault I, Dicaire MJ, Tetreault M, et al. Diversity of ARSACS mutations in French-Canadians. Can J Neurol Sci. 2013; 40:61-66.

6. Takiyama Y. Autosomal recessive spastic ataxia of Charlevoix-Saguenay. Neuropathology. 2006; 26:368-375.

7. Anziska Y, Sternberg A. Exercise in neuromuscular disease. Muscle Nerve. 2013; 48:3-20.

8. Roeder L, Costello JT, Smith SS, Stewart IB, Kerr GK. Effects of resistance training on measures of muscular strength in people with Parkinson's disease: A systematic 
review and meta-analysis. PloS One. 2015; 10:e0132135.

9. Lambert CP, Archer RL, Evans WJ. Muscle strength and fatigue during isokinetic exercise in individuals with multiple sclerosis. Med Sci Sports Exerc. 2001; 33:16131619.

10. Mantria S, Fullarda ME, Dudaa JE, Morley JF. Physical activity in early Parkinson disease. J Parkinsons Dis. 2018; 8:107-111.

11. Logan LR. Rehabilitation techniques to maximize spasticity management. Top Stroke Rehabil. 2011; 18:203211.

12. Stevenson VL. Rehabilitation in practice: Spasticity management. Clin Rehabil. 2010; 24:293-304.

13. Labruyère R, van Hedel HJA. Strength training versus robot-assisted gait training after incomplete spinal cord injury: A randomized pilot study in patients depending on walking assistance. J Neuroeng Rehabil. 2014; 11:4.

14. Reid S, Hamer P, Alderson J, Lloyd D. Neuromuscular adaptations to eccentric strength training in children and adolescents with cerebral palsy. Dev Med Child Neurol. 2010; 52:358-363.

15. Krivickas LS. Exercise in Neuromuscular Disease. J Clin Neuromuscul Dis. 2003; 5:29-39.

16. Richardson D. Physical therapy in spasticity. Eur J Neurol. 2002; 9:17-22

17. Voet NB, van der Kooi EL, Riphagen II, Lindeman E, van Engelen BG, Geurts AC. Strength training and aerobic exercise training for muscle disease. Cochrane Database Sys Rev. 2013; 7:CD003907.

18. Surakka J, Romberg A, Ruutiainen J, Aunola S, Virtanen A, Karppi SL, Mäentaka K. Effects of aerobic and strength exercise on motor fatigue in men and women with multiple sclerosis: A randomized controlled trial. Clin Rehabil. 2004; 18:737-746.

19. Ferraz DD, Trippo KV, Duarte GP, Neto MG, Bernardes Santos KO, Filho JO. The effects of functional training, bicycle exercise, and exergaming on walking capacity of elderly patients with Parkinson disease: A pilot randomized controlled single-blinded trial. Arch Phys Med Rehabil. 2018; 99:826-833.

20. Snider J, Müller ML, Kotagal V, Koeppe RA, Scott PJ, Frey KA, Albin RL, Bohnen NI. Non-exercise physical activity attenuates motor symptoms in Parkinson disease independent from nigrostriatal degeneration. Parkinsonism Relat Disord. 2015; 21:1227-31.

21. Aitkens SG, McCrory MA, Kilmer DD, Bernauer EM. Moderate resistance exercise program: Its effect in slowly progressive neuromuscular disease. Arch Phys Medi Rehabil. 1993; 74:711-715.

22. Adams MM, Hicks AL. Comparison of the effects of body-weight-supported treadmill training and tilt-table standing on spasticity in individuals with chronic spinal cord injury. J Spinal Cord Med. 2011; 34:488-494.

23. Mayer M. Clinical neurokinesiology of spastic gait. Bratisl Lek Listy. 2002; 103:3-11.

24. Adams MM. Assessment and activity-based management of spasticity after spinal cord injury. (Master thesis). McMaster (ON): McMaster University; 2008

25. Dietz V, Harkema SJ. Locomotor activity in spinal cordinjured persons. J Appl Physiol. 2004; 96:1954-1960.
26. Gagnon C, Lessard I, Brais B, Côté I, Lavoie C, Synofzik M, Mathieu J. Validity and reliability of outcome measures assessing dexterity, coordination, and upper limb strength in autosomal recessive spastic ataxia of CharlevoixSaguenay. Arch Phys Med Rehabil. 2018; doi: 10.1016/ j.apmr.2018.01.

27. Schmitz-Hübsch T, du Montcel ST, Baliko L, et al. Scale for the assessment and rating of ataxia: Development of a new clinical scale. Neurology. 2006; 66:1717-1720.

28. Trouillas P, Takayanagi T, Hallett M, et al. International Cooperative Ataxia Rating Scale for pharmacological assessment of the cerebellar syndrome. The Ataxia Neuropharmacology Committee of the World Federation of Neurology. J Neurol Sci. 1997; 145:205-211.

29. Baechle TR, Earle RW, Wathen D. Resistance training. In: Baechle TR, Earle RW (eds): Essentials of strength training and conditioning. 2nd ed. Champaign (IL): Human Kinetics; 2000.

30. Kalinova E, Leone M. Tests d'évaluation de la capacité fonctionnelle chez l'adulte de 55 ans et mieux (Assessment of functional capacity in adults aged 55 and over). Presses de l'Université du Québec, Québec (QC), Canada, 2009. (in French)

31. Noehren B, Abraham A, Curry M, Johnson D, Ireland ML. Evaluation of proximal joint kinematics and muscle strength following ACL reconstruction surgery in female athletes. J Orthop Res. 2014; 32:1305-1310.

32. American Association of Cardiovascular and Pulmonary Rehabilitation. Guidelines for pulmonary rehabilitation programs (2nd ed.). Champaign (IL): Human Kinetics; 1993.

33. Bohannon RW, Andrews AW, Thomas MW. Walking speed: Reference values and correlates for older adults. J Orthop Sports Phys Ther. 1996; 24:86-90.

34. Bui HT, Audet O, Mathieu J, Gagnon C, Leone M. Computer-based assessment of upper-limb incoordination in autosomal recessive spastic ataxia of CharlevoixSaguenay patients: A pilot study. J Neurol Sci. 2017; 380:68-73.

35. Bohannon RW. Muscle strength and muscle training after stroke. J Rehabil Med. 2007; 39:14-20.

36. Kilmer DD. Response to resistive strengthening exercise training in humans with neuromuscular disease. Am J Phys Med Rehabil. 2002; 81:121-126.

37. Allen NE, Sherrington C, Canning CG, Fung VS. Reduced muscle power is associated with slower walking velocity and falls in people with Parkinson's disease. Parkinsonism Relat Disord. 2010; 16:261-264.

38. Toole T, Park S, Hirsch MA, Lehman DA, Maitland CG. The multicomponent nature of equilibrium in persons with parkinsonism: A regression approach. J Neural Transm. 1996; 103:561-580.

39. Gschwind YJ, Kressig RW, Lacroix A, Muehlbauer T, Pfenninger B, Granacher U. A best practice fall prevention exercise program to improve balance, strength/power, and psychosocial health in older adults: Study protocol for a randomized controlled trial. BMC Geriatr. 2013; 13:105.

(Received June 4, 2018; Revised June 27, 2018; Accepted June 29, 2018) 